\title{
Strates
}

STRATES Matériaux pour la recherche en sciences sociales

$12 \mid 2006$

Nouvelles tensions impériales et recompositions en Europe centrale, orientale, et CEI

\section{Des catégories politiques utilisées dans la Russie contemporaine lors du dernier recensement}

Alain BLUM, Catherine GOUSSEFF et Xavier LE TORRIVELLEC

\section{(2) OpenEdition}

Journals

Édition électronique

URL : http://journals.openedition.org/strates/1722

DOI : $10.4000 /$ strates. 1722

ISSN : $1777-5442$

Éditeur

Laboratoire Ladyss

Édition imprimée

Date de publication : 31 décembre 2006

ISSN : 0768-8067

Référence électronique

Alain BLUM, Catherine GOUSSEFF et Xavier LE TORRIVELLEC, « Des catégories politiques utilisées dans la Russie contemporaine lors du dernier recensement », Strates [En ligne], 12 | 2006, mis en ligne le 19 juillet 2007, consulté le 08 septembre 2020. URL : http://journals.openedition.org/strates/1722 ; DOI : https://doi.org/10.4000/strates.1722

Ce document a été généré automatiquement le 8 septembre 2020.

Tous droits réservés 


\title{
Des catégories politiques utilisées dans la Russie contemporaine lors du dernier recensement
}

\author{
Alain BLUM, Catherine GOUSSEFF et Xavier LE TORRIVELLEC
}

Le recensement de 2003 : nouveautés? (Alain Blum)

1 Chaque recensement a toujours été l'objet de débats intenses, très polémiques sur l'usage, la nature des catégories et le moyen de les entériner sur les grilles officielles. La formulation des catégories d'identification collectives a fluctué au cours du siècle car celles-ci ont toujours été fondées sur des déclarations personnelles et marquées par le refus d'une définition extérieure.

2 Dans l'histoire des recensements soviétiques, l'enjeu primordial était celui de la définition des catégories nationales. La liste des nationalités a toujours donné lieu à un débat très riche. Organismes publics et institutions scientifiques ont constitué des listes qui ont abouti à un dictionnaire des nationalités qui offre un panorama de la représentation officielle de la description nationale du territoire, source précieuse de renseignements sur les transformations des conceptions, des formulations et le recodage des différentes nationalités.

3 Le recensement de 1939 essaya de faire coïncider territoire et nationalité, au nom d'une conception plus administrative que scientifique, même si on note la persistance de la catégorie ethnographique. Il est intéressant de voir aujourd'hui comment les catégories anciennement fusionnées puis acceptées... resurgissent dans les perceptions aujourd'hui, et tentent de s'imposer à la place des constructions officielles.

4 Les matériaux conservés par les agents recenseurs permettent de comprendre comment les catégories sont acceptées par les populations. L'enquête qui a été réalisée en 2003 tire son originalité du suivi du recensement sur le terrain : elle a permis de voir comment les enquêteurs réagissent, quels débats sont mis en œuvre, et de dépasser ainsi le cadre formel de la construction des catégories pour mieux comprendre les liens entre la démarche politique, statistique et sociale derrière ces formes d'inscription. 
5 La plus importante innovation depuis la fin de l'URSS a été la suppression de la catégorie nationale pour penser l'État de façon plus homogène. Certaines républiques ont refusé ce retrait comme le Tatarstan. Aussi, dans le recensement de 2003, s'est-on posé la question de savoir s'il fallait maintenir la question de la nationalité, quelle devait être la forme de la question? si elle devait être en continuité avec le passé ? si on devait poser des questions sur les langues maternelles? quelles nationalités reconnaître? si l'on devait poser des questions religieuses?

6 Le pouvoir pouvait craindre, à juste titre, la résurgence de catégories nationales supprimées en 1937 et en 1939, car celle-ci est patente depuis la fin de l'URSS. Si le gouvernement tend à imposer une forme de représentations, les élites locales territorialisées sont néanmoins centrales dans la manière de redéfinir les nouveaux peuples.

7 La seconde innovation importante de ce recensement concerne la volonté de garantir la confidentialité pour protéger la liberté individuelle, notamment en matière fiscale. Ce qui a incité les juristes du gouvernement central à ne pas rendre obligatoire le recensement, ni l'attribution d'une nationalité ; on laissa entendre, au contraire, que l'État n'imposait rien pour trancher avec la période soviétique et par peur d'un échec comme en 1937.

8 Le refus de recensement fut surtout important dans les milieux résidentiels privilégiés inaccessibles et méfiants, vu l'importance des enjeux.

La région de Rostov : modèle de région multiculturelle et lieu emblématique des Cosaques (Catherine Gousseff)

9 L'enquête conduite dans cette région cherchait à savoir comment se reconstituait une région frontière des marches de l'empire russe qui atteste de la présence de nouveaux migrants venus de la CEI et d'une importante communauté cosaque, originaire du Don. La dimension multinationale de la région de Rostov, qui est aussi capitale de la Russie du Sud, est un phénomène historique amplifié par les nouvelles migrations. Celles-ci en font un modèle de coexistence pacifique démontré par plusieurs aspects: l'administration présente cette mosaïque de nationalités comme allant de soi au plan régional chez tous les représentants de la région. Aucun contre-discours n'existe sur la réalité de cette bonne coexistence nationale. Les élus la présentent aussi sous ses meilleurs auspices. Toutes les communautés tiennent également le même discours, mis à part les communautés cosaques. Une partie significative des Cosaques n'est pas enregistrée, en effet, depuis leur entrée officielle dans l'État. La question cosaque est apparue tardivement à travers leurs revendications et tranche par rapport aux relations pacifiques existant entre les autres populations. Après l'été 2002, la grande question fut, dans cette région, l'enregistrement des Cosaques.

Le renouveau cosaque des années 1990

10 Le renouveau de ce groupe a été massif dans ses manifestations sur le territoire de la Fédération de Russie, dans les années 1990. On note l'apparition simultanée des sociétés cosaques, restauration de corps d'armée, très représentées dans les régions du sud de la Russie (le Don, le Kouban et le Terek). Mais, cette explosion dépasse le cadre des implantations historiques de la cosaquerie. En 1997, 438 sociétés cosaques étaient implantées sur plus de $50 \%$ du territoire de la Fédération. Il s'agit d'un phénomène large, difficile à quantifier: l'administration dénombre environ cinq millions de Cosaques en Russie voire six millions et demi à l'échelle de l'ex-URSSS, mais les variations sont importantes selon les interlocuteurs. Ils représentent un poids politique 
important à l'échelle régionale : le recensement est sur ce point plus important pour eux que des élections car il leur fournit enfin une estimation exacte de leur importance numérique, 80 ans après leur stigmatisation.

11 Ce renouveau a pris comme point d'ancrage la loi d'avril 1991 sur les peuples réprimés. Les Cosaques ont justifié leur revendication pour la restauration de leurs droits historiques et un certain nombre de compensations en tant que peuples réprimés en s'appuyant sur elle : leurs revendications sont variables d'une société et d'une région à l'autre. Certaines sociétés ont des revendications modérées. D'autres sont plus radicales: celles du Don sont allées jusqu'à demander le rétablissement de leurs frontières administratives historiques lors du pic du renouveau des année 1992-1993 car celles-ci sont en partie redistribuées entre plusieurs régions, ce qui alimente des menaces de sécessions et des revendications d'autonomie régionale régulièrement agitées par les Cosaques de la région.

Les sociétés cosaques revendiquent leur caractère militaire, leur hiérarchie, leur identité, présentée dès cette époque comme un ethnos: ce qui justifiait la création d'unités régulières de Cosaques, et restaurait un lien entre les Cosaques du Don et ceux du Kouban, ainsi que le label de peuple tel qu'il a existé au sein de l'Empire russe, avant d'être un groupe militaire. Cette exigence s'est focalisée sur l'inscription en tant que Cosaque auparavant en vigueur sur les passeports et qu'ils continuent à revendiquer pour pouvoir se présenter comme tel (mention de la nationalité).

La réponse de l'État a été rapide et cohérente jusqu'à la fin des années 1990 : le premier acte institutionnel a été de réformer en 1993 les structures militaires de Russie du Sud qui marquent une ouverture pour la reconstitution d'une armée avec un statut spécifique pour les Cosaques.

Face à leur exigence de reconnaissance comme peuple, l'État répondit en les décrivant comme un groupe social ou saslov tel qu'il a été défini sous l'Empire russe (cette catégorie définissaient un type d'activité et des droits juridiques affiliés c'est-à-dire une modalité d'existence de la cosaquerie au sein de l'État russe avec le privilège historique de garde-frontière dans des régions mal contrôlées par l'Empire). Cette réponse qui leur reconnaît un droit légitime de communauté culturelle et historique fondée sur la saslovie, et non celui de peuple, leur donnait le droit de revendiquer ce statut. C'est avant tout une décision pragmatique pour prévenir le risque de constitution de groupes militaires non contrôlés par l'État.

La loi de 1993 a ouvert à une série de mesures qui ont abouti en 1996, avant les élections présidentielles, à la création d'une administration centrale cosaque auprès de la Fédération de Russie, première représentation d'un statut avec une attribution spécifique significative de leur intégration au service de l'État. Les décrets cadres en 1995 ont défini les modalités d'existence de cette administration basées sur le principe d'enregistrement des sociétés cosaques, moment test pour voir quelle pouvait être la réaction des Cosaques face à ce processus d'institutionnalisation au sein de l'État russe. La réticence a été très forte. De fortes divisions sont apparues à propos de l'enregistrement des sociétés cosaques. Depuis 1995, les deux attitudes coexistent, induisant une grande hétérogénéité des discours et une certaine confusion. Ce mouvement de résistance à l'enregistrement s'explique en partie par une confusion entre pouvoir politique et État russe, et en partie par la méfiance liée à un passé lourd de répression et de mise sous tutelle et la crainte d'un retournement de situation qui entraînerait de nouvelles formes de répression. 

Cosaques furent considérés comme peuple et réprimés comme tels dans les grandes répressions des années 1930 dont 1936-1937. L'aspect instrumental de la compensation n'existe pas seulement chez eux, mais chez tous les autres groupes perçus comme peuples au moment des répressions qui s'appuient aujourd'hui sur la loi pour justifier ces revendications.

La spécificité bachkire à travers le recensement (Xavier Le Torrivellec)

21 l'identité ethnique différente des Tatars et des musulmans est mise en avant par Lénine qui double le territoire et la population des Bachkirs pour contrer l'hégémonie tatare et fait passer celle-ci d'un statut de minorité au statut de majorité en 1922. De cette époque, date le ressentiment des Tatars à l'égard des Bachkirs, amplifié par la politique d'indigénisation qui a privilégié les fonctions représentatives de l'ethnie éponyme, en facilitant la création d'une culture, d'une littérature bachkires. Entre 1939 et 1979, on note l'importante croissance des recensés comme Bachkirs. L'imposition de l'identité 
bachkire a préservé celle-ci dans le Sud de la république, moins touché par les migrations (la population toujours mélangée parlant majoritairement le tatar a toujours fluctué entre deux identités, de nationalité bachkir et de langue maternelle, tatare). En 1970, on imposa le bachkir au Nord-Ouest pour faire correspondre langue et identité et en finir avec les faux Bachkirs. Cette problématique garde une grande actualité : en 1990, où cette politique aboutit à la déclaration de souveraineté et à la création d'un quasi-État national qui profite de la faiblesse de l'État eltsinien pour trouver une légitimation. L'arrivée de Vladimir Poutine provoque une rivalité de plus en plus affirmée entre les républiques du Tatarstan et de Bachkirie.

Le recensement initié par le pouvoir central qui vise à réaffirmer son autorité sur l'ensemble du territoire donne aussi l'opportunité à la société d'affirmer son existence. Les autorités locales voulurent faire de ce recensement un moment important de légitimation (organisant un concours pour récompenser la meilleure campagne de recensement, ainsi que le travail de chaque district avec une répartition des compétences et du travail à effectuer).

Il fut l'occasion d'une mobilisation virulente, et objet de mémoire dans le cadre du conservatisme local où le passé sert de référence. Les précédents recensements de 1979 et de 1989 ont servi de référence aux mouvements nationaux bachkirs, pour mettre en scène leur égalité avec les Tatars. Côté tatar, celui de 1989, plus démocratique, a permis un retour des populations du Nord-Ouest à une identité originelle tatare; en janvier 2002, eut lieu une forte campagne de persuasion pour démontrer les racines bachkirs ou tatares des populations indécises de cette région.

24 À travers les débats nationalistes entre Bachkirs et Tatars a pu s'esquisser un mouvement d'appropriation individuelle des choix identitaires: le mouvement kriachen ${ }^{1}$ de Tatars convertis au christianisme est exemplaire à cet égard. Chacun semble avoir sa théorie, sa langue ou sa religion sans critères ethniques spécifiques, affirmant plus une volonté individuelle qui rejoint la volonté fédérale d'imposition de valeur libérale à travers le recensement.

L'Altaï : ethnie et représentation identitaire (Alain Blum)

25 Dans l'Altaï montagneux, le recensement fut prétexte à un débat sur le découpage de six peuples turcophones situés dans des villages difficiles d'accès: débat qui met l'accent sur l'inadéquation du cadrage ethnographique avec les expressions des identités. La grille ethnique ne correspond pas aux régions turcophiles où domine la structuration en lignages transversaux qui ont des rameaux dans l'Altaï, mais aussi en Chine et au Kazakhstan.

Le recensement en 1897 des lignages s'avérait plus pertinent. La société fonctionnerait de façon exogame à travers le lignage, et non pas à travers le peuple. Considéré comme une forme d'expression communautaire des élites, le nom du lignage n'est pas donné dans le recensement. En 1999, l'État offrit une certaine reconnaissance qui donna aux habitants de ces villages certains privilèges sur leurs terres (inaliénabilité), pour couper court à leurs revendications. Les débats contemporains renvoient néanmoins à ceux du $\mathrm{XIX}^{\mathrm{e}}$ siècle et délégitiment la représentation ethnographique que Moscou a construit de ces peuples, remettant en cause la volonté d'homogénéisation des représentations identitaires de l'État russe. 


\section{NOTES}

1. Les Kriachen forment une des différentes entités ethniques tatares comme les Tatars Michars, les Tatars de Kazan ou les Tatars de Kasimov. Mais les musulmans donnent le nom de Kriachen à tous les Tatars de la communauté orthodoxe tatare.

\section{RÉSUMÉS}

Les nouvelles divisions territoriales ont été mises à l'épreuve récemment par le dernier recensement qui montre que nombre de catégories ethniques supprimées lors du recensement de 1939 pour simplifier la carte ethnique, ont tendance à réapparaître à la faveur de la loi pour les petits peuples. On y lit toute la complexité d'une structure que le centre veut figer une fois pour toutes dans des catégories stables pour les consolider, mais dans lesquelles certaines populations ne se reconnaissent absolument pas et revendiquent un autre statut, car à tout statut correspond des droits afférents. La bataille pour la nationalité et le code des Cosaques met ainsi en relief les enjeux autour de ces appellations et identifications depuis les récentes constitutions.

Alain Blum, Catherine Goussef, Xavier Letorivellec. Polical categories used in the last census in Russia The new territorial divisions have recently been put to the test by the last census which showed that a number of ethnic groups suppressed during the 1939 census in order to simplify the map, tend to reappear following the law benefiting small ethnic groups. One can read in this all the complexity of a structure which the centre wants to freeze once and for all into stable categories aiming to consolidate these groups but in which some populations do not recognise themselves at all, preferring to claim another status, since there is a corresponding right for each status. Thus the battle for nationality and the Cossacks' Code bring to the fore the stakes which are associated with these labels and identifications since the recent constitutions were written.

\section{AUTEURS}

\section{ALAIN BLUM}

Directeur d'études à l'EHESS, directeur du CERCEC (EHESS/CNRS), blum@ined.fr

CATHERINE GOUSSEFF

Chercheur, Centre du monde russe, EHESS, gousseff@ehess.fr

\section{XAVIER LE TORRIVELLEC}

Docteur de l'EHESS en histoire et civilisations, Ater Inalco, xavierlt@libertysurf.fr 